\title{
New Approaches for the Detection of Minimal Residual Disease in Acute Myeloid Leukemia
}

\author{
Anna van Rhenen, MD, Bijan Moshaver, Gert J. Ossenkoppele, MD, PhD, \\ and Gerrit Jan Schuurhuis, PhD
}

\author{
Corresponding author \\ Gerrit Jan Schuurhuis, PhD \\ VU University Medical Center, CCA 4.24, De Boelelaan 1117, \\ 1081 HV Amsterdam, The Netherlands. \\ E-mail: gj.schuurhuis@vumc.nl
}

Current Hematologic Malignancy Reports 2007, 2:111-118

Current Medicine Group LLC ISSN 1558-8211

Copyright (C 2007 by Current Medicine Group LLC

The detection of minimal residual disease (MRD) in patients with acute leukemia has been studied for about 15 years by different groups in both the United States and Europe. It has been found that MRD detection can be performed using molecular and immunophenotypic aberrancies that are present in the leukemic clone at diagnosis and not in normal bone marrow. When performing MRD assessments after chemotherapy, it is possible to identify patients at risk for relapse. This review is not an overview of all MRD studies, but rather discusses the possibilities for optimizing MRD detection, the use of flow cytometry versus polymerase chain reaction techniques, and the implications for future patient treatment. When informative, we compare literature on MRD in acute myeloid leukemia (AML) with information from MRD studies in acute lymphoblastic leukemia. Finally, we address the promising detection of AML stem cells, the likely cells of origin in $\mathrm{AML}$, for prediction of clinical outcome and guidance of future therapies.

\section{Introduction}

Acute myeloid leukemia (AML) is a malignant disease with an age-adjusted incidence of 3.7 per 100,000 men and women per year in the United States [1]. The disease affects both children and adults. Although about $80 \%$ of adult patients achieve complete remission after intensive chemotherapy, only $30 \%$ to $40 \%$ of patients survive 5 years after diagnosis [2]. Many patients experience a relapse, which is caused by the presence of minimal residual disease (MRD) and in most cases is incurable. There are several hypotheses as to why patients develop MRD after successful initial response to chemotherapy. The primary reason for treatment failure in AML is cellular drug resistance from both drug efflux and apoptosis-related mechanisms, including upstream aberrant signal transduction. Pharmacologic resistance also may play a role.

Resistance mediated by drug efflux occurs in AML via high expression of the multidrug resistance (MDR1) gene (P-glycoprotein [Pgp]) and drug resistance-related proteins MRP1 and LRP and has been shown to predict poor prognosis [3]. Moreover, patients with a multidrugresistant profile at diagnosis have higher MRD frequencies after chemotherapy than patients with a more sensitive phenotype with regard to Pgp [4]. Apoptosis resistance is also important for the development of MRD: high Bcl-2 and/or low Bax expression at diagnosis have been shown to predict poor survival [5]. Moreover, such an apoptosis-resistant profile is correlated with higher levels of MRD after chemotherapy [6•]. Recently the bone marrow microenvironment has attracted a lot of attention as a key player in modulating drug resistance in AML. It has been shown that adhesion through integrin engagement triggers chemotherapy resistance of AML blasts [7]. Moreover, blockade of the Wnt pathway, an important signal transduction pathway in stem cells, by Wnt antagonists supports drug resistance of AML [8]. It is likely that these mechanisms also contribute to the presence of MRD after chemotherapy.

Pharmacologic resistance may play a role when a large tumor load is present, as has been shown for daunorubicin, but the clearance rate of the drug and the cytotoxic activity of the major metabolites also may be important $[9,10]$.

Finally, the mechanisms mentioned above probably contribute to the survival of the leukemic stem cells (LSCs), the supposed cells of origin [11-13]. Hypothetically, LSC regrowth leads to the presence of MRD and subsequently to relapse $[14 \bullet \bullet]$.

\section{MRD Detection Methods}

In patients with acute leukemia, detection and quantification of malignant cells after chemotherapy in remission bone marrow (BM) provides powerful prognostic infor- 


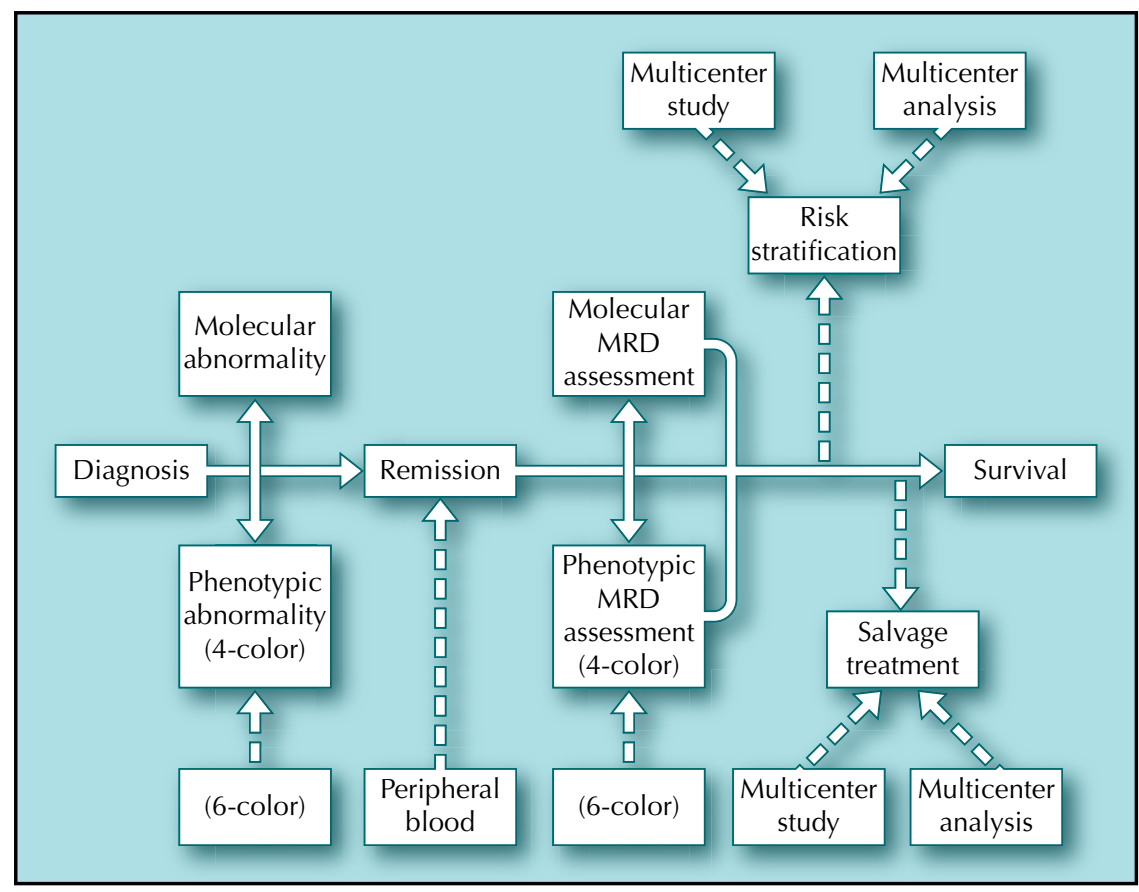

Figure 1. Overview of new approaches for the detection of minimal residual disease (MRD), showing established relationships (solid lines) and relationships under investigation (dashed lines). Molecular or phenotypic abnormalities at diagnosis are used to detect MRD in bone marrow after chemotherapy. The amount of MRD has an impact on survival of patients. New approaches include the use of six-color polychromic flow cytometry instead of four-color multiparameter flow cytometry, the use of peripheral blood instead of bone marrow for MRD assessments, and the clinical decisions that can be made after MRD assessment, including risk stratification and/or salvage therapy, in cases of increasing MRD frequency.

mation for the identification of patient risk categories. This knowledge is expected to facilitate the early detection of impending relapse, may result in risk-adapted therapies, and may offer a short-term endpoint to assess the effectiveness of new, targeted therapies.

If the leukemic cell at diagnosis carries an antigenic or molecular marker that distinguishes it from its normal counterpart, this marker can be used after chemotherapy to detect residual malignant cells in BM. This allows the application of two sensitive methods to identify MRD [15] in both acute lymphoblastic leukemia (ALL) and AML: multiparameter flow cytometry (MFC) and polymerase chain reaction (PCR)-based techniques. Each method has its advantages and disadvantages for the detection of MRD.

The clinical importance of MRD detection and quantification in adult [16] and childhood [17,18] ALL is already established and has been incorporated in pediatric ALL trials. Although a number of studies have reported on the clinical value of MRD detection in AML in children $[19,20]$ and adults $[21,22,23 \bullet, 24 \bullet]$, the information provided is still somewhat limited, compared with what is known for ALL. We will discuss primarily flow cytometry and also PCR-based strategies for the investigation of MRD in patients with AML. A schematic overview of new approaches for the detection of MRD is shown in Figure 1. Finally, we will discuss a new area in MRD, AML stem cells in remission.

\section{Multiparameter flow cytometry}

Immunophenotypical analysis of residual leukemic cells using MFC is an attractive approach for MRD detection: it is sensitive (cell frequency of $10^{-3}$ to $10^{-5}$ ), fast, quantitative, relatively cheap, and, importantly, applicable to more than $90 \%$ of patients with AML, many more than PCR-based MRD detection $[21,22,23 \bullet, 24 \bullet, 25]$. In contrast, both immunophenotypical and PCR-based MRD targets are present in the vast majority of patients with ALL. Applying quadruple marker combinations, MFC uses the presence of aberrant expression of markers, usually referred to as leukemia-associated immunophenotypes (LAIPs or LAPs) [25]. LAPs are present on all leukemic cells or on a subset of them, but they are absent or very infrequent on normal BM cells. These LAPs result from cross-lineage antigen expression, antigen overexpression, antigen underexpression, asynchronous antigen expression, and abnormal light scatter pattern [25]. Several studies have shown that MRD detection based on MFC analysis provides strong prognostic information in AML after both induction and consolidation therapy $[19-22,23 \bullet, 24 \bullet]$.

Unfortunately, in line with the immunophenotypical heterogeneity of AML cells at diagnosis, the LAPs are usually not expressed on the whole blast cell population [23•]. (In ALL, on the other hand, the cell population is immunophenotypically homogenous [17].) The heterogeneity of AML cells affects the sensitivity of the approach, introducing patient-dependent sensitivity. Other limitations to the applicability of MFC in MRD studies include the occurrence of major or minor phenotypic shifts at relapse, potentially resulting in false negativity $[23 \bullet, 26]$; background staining of the normal bone marrow, which is LAP-dependent and strongly affects the specificity [27]; and the absence of LAPs in some patients.

\section{Polychromatic flow cytometry}

MFC techniques are being optimized by technologic advances in new fluorochromes, novel hardware capable 
of measuring more fluorescence parameters, and new software tools capable of analyzing, managing and reducing the large and complex data sets. Thus, polychromatic flow cytometry (PFC) for the detection of different fluorochromes (using more than four colors) offers new possibilities for MRD detection [28].

One of the key issues in MRD monitoring is specificity. Although four-color flow cytometry is commonly used to perform MFC-based MRD detection, monitoring of MRD in patients with AML with a LAP that has relatively high expression on normal BM counterpart cells would benefit from a more specific approach. The inclusion of additional markers might better exclude normal BM cells, resulting in higher specificity. Using such an approach, it has been shown that five-color PFC improves the specificity and reproducibility of MRD monitoring in AML [29]. The other advantages of PFC would be to combine different four-color LAPs in one newly established composite LAP, thereby reducing the amount of BM necessary, the number of tubes, costs, and time.

\section{Polymerase chain reaction}

An increasing number of studies have shown the clinical importance of PCR-based MRD detection in AML [30-32]. Real-time quantitative reverse transcriptase PCR (qRT-PCR) is the most sensitive molecular MRD detection method used in recent years in AML [31,32]. It permits absolute quantification of target DNA or mRNA, in contrast to end-point quantification of the more classic PCR method. The qRT-PCR technique is based on genetic aberrations such as mutations and fusion genes, which occur in subgroups of AML. Recent and future advances in identifying new AMLassociated genetic aberrations eventually may provide such molecular targets for the majority of patients. At diagnosis, quantitative PCR (qPCR) enables the assessment of the transcriptional ratio and the kinetics of the reduction of the leukemic clone; it offers the possibility of early detection of a recurring clone. Although the qRT-PCR method may be complex and time-consuming, it is highly sensitive and has been applied for the quantification of fusion transcript levels before, during, and after therapy.

\section{Molecular targets for PCR-based MRD monitoring}

The most common rearrangements producing fusion genes in AML are $\mathrm{t}(8 ; 21), \mathrm{t}(15 ; 17)$, and inv(16)/ $\mathrm{t}(16 ; 16)$, which occur in a minority $(9 \%$ to $19 \%)$ of all patients with AML and have a favorable prognosis [33]. The corresponding fusion transcripts AML1-ETO, PML-RARA, and CBFB-MYH11 can be detected by qRT-PCR at diagnosis and follow-up to identify MRD [34]. Other potential targets for PCR-based MRD detection in AML are mutations in FLT3, MLL, and NPM1, and overexpression of WT1, EVI1, and PRAME. The usefulness of these targets for MRD detection is pres- ently under investigation either by single institutions or larger networks.

\section{FLT3 mutations}

The presence of internal tandem duplications within the FLT3 gene (FLT3-ITD) characterizes an unfavorable subset of the cytogenetically defined intermediate-risk group. These mutations can be detected in about $20 \%$ to $30 \%$ of patients with AML [35]. Previous studies showed that not all analyzed patients maintained the same FLT3 status at relapse and therefore the results of follow-up analysis should be evaluated with special care; some patients relapse with a leukemic clone that lacks the mutation [36].

\section{MLL-PTD mutations}

The frequency of partial tandem duplications of the $M L L$ gene (MLL-PTD), which are indicative of an unfavorable outcome, is $3 \%$ to $4 \%$ at diagnosis and up to $10 \%$ in AML with normal karyotype. It has been shown that MRD monitoring is possible using these $M L L$ aberrations [37].

\section{NPM1 mutations}

Mutations of the nucleophosmin member 1 (NPM1) gene occur predominantly in patients who have AML with normal karyotype; it has been reported to occur in $46 \%$ to $62 \%$ of these cases [38]. A recent study suggested that qPCR assays for NPM1 mutations is suitable for monitoring and quantifying MRD in patients with AML and normal karyotypes [39].

\section{WT1 overexpression}

The Wilms tumor gene (WT1) encoding a zinc finger transcription factor is a tumor suppressor gene that functions as a potent transcriptional repressor of several growth factors. Overexpression is related to the maintenance of the viability of leukemic cells through regulation of $\mathrm{Bcl}-2$ expression [40]. WT1 overexpression can be detected in almost all AML patients, and high levels of WT1 after chemotherapy are predictive for survival [41,42]. Recently the MRD Working Party of the European Leukemia Network (www.leukemianet.org) decided to explore the possibilities of WT1 as an MRD marker.

\section{EVI1 overexpression}

The EVI1 gene, which is associated with rearrangements of $3 \mathrm{q} 26$, is overexpressed in $20 \%$ of patients with newly diagnosed AML and is associated with poor prognosis [43]. To our knowledge, no studies on the use of EVI1 overexpression as a marker for MRD have been published.

\section{PRAME overexpression}

Overexpression of the preferentially expressed antigen of melanoma (PRAME) gene has been found in $62 \%$ of 
children with AML and is correlated with a favorable outcome [44]. A good correlation between PRAME mRNA expression after chemotherapy and disease status has been found [45].

\section{Comparison of MFC and qRT-PCR}

It is important to compare the value for patient prognosis of quantifications of MRD based on qRT-PCR and MFC. Several reports using patients with ALL showed generally concordant results $[46,47]$, but few data are available on this issue in AML. A recent study has shown high correlations between PCR-based and MFC-based MRD detection and recommends the combined use of both techniques [48]. Although qRT-PCR is more sensitive than MFC in quantifying MRD in patients with translocations, it is unknown how many patients can be subjected to PCRbased strategies using all the new targets that have been identified, as these have not been studied together. Further prospective studies are needed to define the best approach for MRD monitoring in AML patients.

\section{Blood versus bone marrow for MRD measurements}

In all MRD studies in patients with AML, measurements are performed on BM samples. It would of course be convenient for both patient and doctor to use peripheral blood (PB) instead, but only one relevant study has been performed in AML [49]. This study described the clearance of blasts from the $\mathrm{PB}$ at days 1 to 8 after the start of chemotherapy, which correlated with MRD frequency in the $\mathrm{BM}$ at day +14 . Clearly more studies are necessary.

Regarding molecular MRD assessments, Goulden et al. [50] reported results of the Medical Research Council AML 15 trial in patients with acute promyelocytic leukemia. It was found that BM is more reliable than PB for the detection of PML-RAR $\alpha$. For $t(8,21)$, only one study in pediatric AML showed that PB was equal to BM [51]. For ALL, the use of PB instead of BM has been extensively studied. It was concluded that BM samples cannot easily be replaced by PB sampling for MRD analysis in childhood precursor-B-ALL, in line with the BM origin of this disease [52,53]. In T-ALL, which is of thymic origin, BM sampling might be replaced by $\mathrm{PB}$, probably because the dissemination of T-ALL cells to BM and PB is comparable [53]. Because AML is also of BM origin, it may not be possible for $\mathrm{PB}$ to simply replace BM. At present, there is not enough evidence that $\mathrm{PB}$ sampling can replace $\mathrm{BM}$, except possibly for AML M3.

\section{Multicenter MRD Analysis and Prospective Studies}

The ultimate goal of MRD studies is implementation in clinical practice for both risk stratification and guidance of clinical intervention. To this end, consensus in MRD analysis is necessary and guidelines should be published enabling uniformity of all procedures, thereby allowing
MRD analysis in multiple centers. In The Netherlands and Belgium, a working group, the Dutch/Belgium Task Force for MRD detection in AML, composed of several academic centers in cooperation with the European Society for Clinical Cell Analysis (ESCCA), has been initiated to prepare MRD guidelines. Recently, consensus has been reached on the analysis of AML samples in the diagnostic phase and end terms have been determined (Feller, Unpublished data). The next target is to achieve consensus regarding analysis in the MRD phase, based on the end terms defined for the diagnostic phase. This effort ultimately should result in consensus guidelines for MRD analysis that can be applied in different centers.

To definitely prove the value of MRD measurements, prospective studies are needed to answer two important questions: 1) Can MRD assessment identify high-risk patients within both the standard-risk and good-risk groups, who require additional or more intensive therapy regimens? 2) Can MRD assessment identify those at very low risk of relapse, who might be candidates for reduction of treatment? The Dutch hemato-oncology association, the HOVON, has recently started the HOVON-42A protocol for the treatment of patients with AML who are aged under 60 years. In this multicenter, prospective, randomized study, patients are treated with two induction courses and one consolidation course. One of the major side studies is a prospective study on the value of MRD assessments using MFC. BM samples are collected for both MFC and PCR, allowing a retrospective comparison between molecular and immunophenotypical MRD. The HOVON study 81 also will include BM sampling for MRD studies in patients aged 60 or older. St. Jude Children's Research Hospital in Tennessee, USA, has already performed a prospective single-center study of flow cytometry in AML with a relatively small number of children [20].

Multicenter trials are needed for stratification of AML patients based on their MRD frequency, to see whether patients can benefit from reduced or intensified treatment regimens. For acute promyelocytic leukemia, studies have been published on salvage therapy. Preliminary evidence from the GIMEMA group suggests a survival benefit for pre-emptive therapy (at a stage of molecularly resistant disease) when compared with treatment at the point of relapse [54]. This shows that pre-emptive therapy is potentially beneficial. For comparison, many studies in pediatric ALL patients in both the United States and Europe have already included MRD frequency assessments as a risk-stratification step with consequences for therapy. Regarding adult patients, in the German Multicenter ALL (GMALL) trial, salvage treatment is intended to be started at the time of recurrence of quantifiable MRD.

\section{MRD as a short-term endpoint in clinical studies}

In recent years, many new targets have been identified that need testing in clinical practice. However, the study 
designs that are generally used require the accrual of many patients and a long follow-up. Short-term endpoints would be of great importance to evaluate new randomized phase II trials with new treatment modalities. Because MRD reflects the sum of all parameters contributing to quality of response to therapy, it might serve as an early endpoint to assess effectiveness of new therapeutic modalities. The correlation of multidrug resistance [4], apoptosis [6•], and FLT3-ITD [55] at diagnosis with MRD frequencies indicates that such an approach is very promising.

\section{Stem Cells and MRD CD34+CD38-}

AML is generally regarded as a stem cell disease. In CD34-positive AML, the stem cell has been recognized as CD38-negative [11]. It is also generally thought that LSCs preferentially survive chemotherapy and grow out to cause the presence of MRD, which leads to relapse of the disease in some cases. If so, stem cell characteristics at diagnosis should show a correlation with MRD cell frequency after chemotherapy, which indeed was found for the frequency

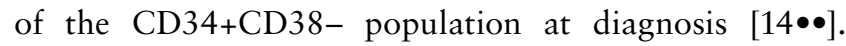
Because MRD frequency shows a strong correlation with survival $[21,22,23 \bullet, 24 \bullet]$, we hypothesized that the CD34+CD38- cell frequency itself at diagnosis should be directly correlated with the survival of patients, and this

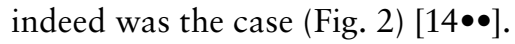

Similar to the characterization of the leukemic blasts using LAP expression, the identification of immunophenotypical characteristics specific for the malignant CD34+CD38- cells at diagnosis would offer opportunities to study the stem cell compartment after chemotherapy. This information in turn would enable not only identification of patients at risk for relapse, but also the characterization of these cells, which could then help to identify new targets for therapy. One of the possible markers, CD123, is expressed on most AML blasts and on the leukemic CD34+CD38subpopulation at diagnosis; it is reported not to be present on normal CD34+CD38- cells [56]. Also CD33, a common myeloid antigen, is present on both the majority of the blasts and on the CD34+CD38- cells. Recently, however, Taussig et al. [57•] reported that both CD33 and CD123 were present not only on malignant CD34+CD38- cells but also on the normal BM CD34+CD38- counterpart. We obtained similar results, especially for BM regenerating after chemotherapy (van Rhenen, Unpublished data). Thus, neither CD123 nor CD33 may be suitable to specifically detect malignant CD34+CD38- cells in the BM of patients with AML during or after chemotherapy.

Therefore the search for markers that are specifically expressed on AML stem cells but not on normal stem cells is ongoing. Recently we have found that C-type lectinlike molecule-1 (CLL-1) may represent such an antigen. CLL-1 expression on AML blasts is comparable to CD33 expression [58 ${ }^{\bullet}$ and, moreover, is present on malignant

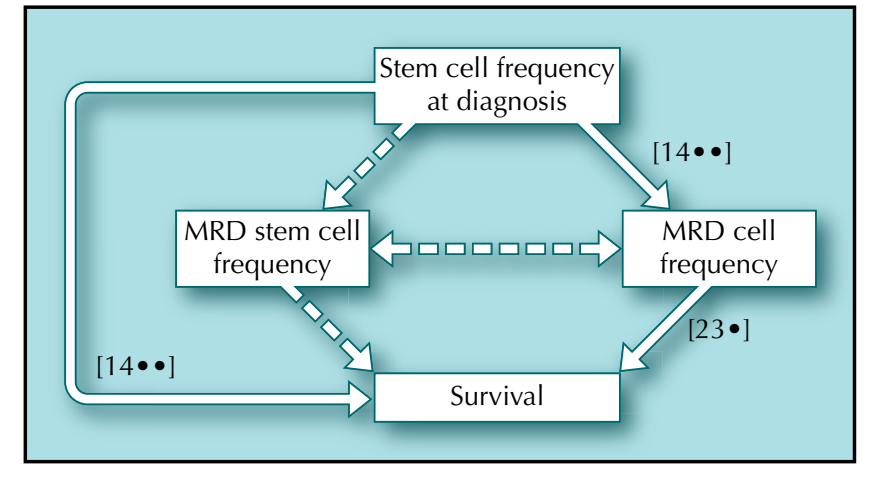

Figure 2. This figure shows the interrelationships between stem cells (CD34+CD38-), frequency of minimal residual disease (MRD), and patient survival. Solid lines represent established relationships and dashed lines represent possible relationships that are under investigation. At diagnosis, stem cell frequency can be determined, which correlates with both MRD frequency after chemotherapy $[14 \bullet \bullet]$ and survival $[14 \bullet \bullet]$. MRD frequency itself is related to survival $[23 \bullet]$ and also to the MRD stem cell frequency, which in turn may have an impact on survival of patients. It remains to be established whether these results are also true for the leukemic stem cell side population.

CD34+CD38- cells in the majority of patients with AML who are positive for CLL-1 at diagnosis [59]. In contrast to CD123 and CD33, normal CD34+CD38- cells lack CLL1 expression under all conditions of disease (van Rhenen, Unpublished data). In addition (and unexpectedly), many lineage-associated antigens, which make up most of the LAPs already discussed, were found to be prominently present on the AML CD34+CD38- compartment but not on the steady state and regenerating normal bone marrow CD34+CD38- counterpart [60]. The lineage markers thus can be used for the detection of residual malignant CD34+CD38- cells in BM during or after therapy.

In conclusion, specific detection of malignant CD34+CD38 - cells after chemotherapy is possible using CLL-1 and lineage marker expression, but it probably is not possible using the presumed LSC marker CD123 or CD33. CD33 is the target of gemtuzumab ozogamicin (Mylotarg ${ }^{\circledR}$, Wyeth Pharmaceuticals, Philadelphia, PA), presently used in the clinic. The presumed expression of CD33 on normal stem cells likely offers part of the explanation for the toxicities seen with this therapy.

\section{Side population}

The CD34+CD38- compartment does not provide the stem cell compartment for all patients with AML. At diagnosis, $5 \%$ of patients with AML who have a CD34+ phenotype lack a detectable CD34+CD38- compartment $(<0.01 \%)$ and about $20 \%$ are CD34 negative and thereby, by definition, CD34+CD38- negative [14••]. An alternative stem cell compartment for these patients is offered by the so-called side population (SP). A decade ago, Goodell et al. [61] reported the presence of an extremely small but distinct population of cells in normal BM of mice, capable of efficient Hoechst 33342 dye efflux. This population was highly enriched for hematopoietic stem 
cells (HSCs). This HSC population was identified too in human BM and shown to be largely CD34 negative with a high repopulation potential and successful engraftment in mice [62]. SP cells are also present in BM obtained from patients with AML, and these were capable of initiating leukemia after transplantation into NOD/SCID mice, suggesting that these cells might be candidate LSCs [63]. Moreover, AML-specific cytogenetic abnormalities were present in SP cells obtained both at diagnosis and after chemotherapy in remission BM. These findings suggested that SP cells in normal BM may be a target for leukemic transformation.

The immunophenotypical characteristics of SP cells have not yet been analyzed in great detail. Both CD34 and CD38 showed a large range of expression with large interindividual differences. The precise relationship between SP cells and the antigenic marker-defined CD34+CD38subset is unknown. Of interest, the SP cell frequency is much lower than the CD34+CD38- cell frequency (in our hands, median $0.02 \%$ vs $0.44 \%$ of white blood cells). The immunophenotype of AML SP cells, in addition to being reported as CD34+CD38- or CD34-CD38-, may also be CD34+CD38+ [64].

SP cells may be partly or completely positive for the lineage markers CLL-1, CD123, or both, strongly suggesting that most SP cells represent the malignant clone, which in a few cases could be confirmed by FISH analysis [65]. Furthermore, SP cells from normal BM and control regenerating $\mathrm{BM}$ were completely negative for CLL-1 and the lineage markers, but not for CD123. Similar to the CD34+CD38-, using these discriminative markers, SP cell frequency after chemotherapy paralleled normal ("whole blast") MRD and clinical outcome [66].

Thus, SP cells indicating AML can be discriminated from normal SP cells based on the expression of CLL-1 and lineage markers, and these markers can be used for the detection of MRD SP cells in BM after therapy. Future studies using PFC, which enables the use of Hoechst staining together with multiple marker combinations, will elucidate the relationship between the SP stem cell compartment and the CD34+CD38- stem cell compartment.

Clinical implications of MRD stem cell detection in AML Recent insights into the nature of normal and malignant stem cells have led to the identification of quiescent and drug-resistant LSCs as likely candidates responsible for relapse $[11,14 \bullet \bullet]$. To predict impending relapses prior to clinical manifestations and to manage patient-adapted postremission therapies, it may be of great importance to detect MRD stem cells after chemotherapy using CD34+CD38- or SP AML phenotypes, besides detecting MRD using the whole blast compartment. Evidence for this role comes from the observation that AML stem cell parameters offer prognostic information additional to MRD frequency assessment $[60,66]$.
Apart from such clinically applicable prognostic information, the flow cytometric approaches detect viable malignant CD34+CD38- and/or SP populations, thereby allowing further characterization of these cells with the aim of developing new therapies directed at stem cells or applying present therapies in a more patienttailored way. Moreover, as interaction of LSCs with the BM microenvironment, the stem cell niche, is important for their survival $[11,14 \bullet \bullet]$, future studies should also focus on the molecular mechanisms involved in the interactions between the stem cell niche and the LSCs to determine the therapeutic windows for new anticancer therapies directed at LSCs in a clinical setting $[11,14 \bullet \bullet]$.

\section{Conclusions}

MRD detection in AML has shown prognostic significance. Immunophenotypical and PCR-based methods should be compared, including all new targets that are available for PCR-based strategies. PFC might enable more specific analysis than MFC. The preferential study material is still BM. After prognostic MRD studies, it may become possible to apply MRD monitoring to clinical decision making. The introduction of stem cells in MRD studies may further improve the clinical significance of these studies and may reveal the most relevant target-cell population for the design of new therapies.

\section{Acknowledgment}

The first two authors, Anna van Rhenen and Bijan Moshaver, contributed equally to the writing of this article.

\section{References and Recommended Reading}

Papers of particular interest, published recently, have been highlighted as:

- Of importance

-. Of major importance

1. Ries LAG, Harkins D, Krapcho M, et al.: SEER Cancer Statistics Review, 1975-2003. Bethesda, MD: National Cancer Institute; 2006. Available at http://seer.cancer. gov/csr/1975_2003/

2. Lowenberg B, Griffin JD, Tallman MS: Acute myeloid leukemia and acute promyelocytic leukemia [review]. Hematology Am Soc Hematol Educ Program 2003, 82-101.

3. Legrand O, Simonin G, Perrot JY, et al.: Pgp and MRP activities using calcein-AM are prognostic factors in adult acute myeloid leukemia patients. Blood 1998, 91:4480-4488.

4. van der Pol MA, Feller N, Ossenkoppele GJ, et al.: Minimal residual disease in acute myeloid leukemia is predicted by P-glycoprotein activity but not by multidrug resistance protein activity at diagnosis. Leukemia 2003, 17:1674-1677.

5. Del Poeta G, Venditti A, Del Principe MI, et al.: Amount of spontaneous apoptosis detected by $\mathrm{Bax} / \mathrm{Bcl}-2$ ratio predicts outcome in acute myeloid leukemia (AML). Blood 2003, 101:2125-2131. 
6.• van Stijn A, Feller N, Kok A, et al.: Minimal residual disease in acute myeloid leukemia is predicted by an apoptosis-resistant protein profile at diagnosis. Clin Cancer Res 2005, 11:2540-2546.

An apoptosis-resistant profile at diagnosis, studied using flow cytometry, predicts the presence of high MRD frequency after chemotherapy.

7. Matsunaga T, Takemoto N, Sato T, et al.: Interaction between leukemic-cell VLA-4 and stromal fibronectin is a decisive factor for minimal residual disease of acute myelogenous leukemia. Nat Med 2003, 9:1158-1165.

8. De Toni F, Racaud-Sultan C, Chicanne G, et al.: A crosstalk between the Wnt and the adhesion-dependent signaling pathways governs the chemosensitivity of acute myeloid leukemia. Oncogene 2006, 25:3113-3122.

9. Kokenberg E, Sonneveld P, Sizoo W, et al.: Cellular pharmacokinetics of daunorubicin: relationships with the response to treatment in patients with acute myeloid leukemia. J Clin Oncol 1988, 6:802-812.

10. Gessner T, Preisler HD, Azarnia N, et al.: Plasma levels of daunorubicin metabolites and the outcome of ANLL therapy. Med Oncol Tumor Pharmacother 1987, 4:23-31.

11. Bonnet D, Dick JE: Human acute myeloid leukemia is organized as a hierarchy that originates from a primitive hematopoietic cell. Nat Med 1997, 3:730-737.

12. Costello RT, Mallet F, Gaugler B, et al.: Human acute myeloid leukemia CD34+/CD38- progenitor cells have decreased sensitivity to chemotherapy and Fas-induced apoptosis, reduced immunogenicity, and impaired dendritic cell transformation capacities. Cancer Res 2000, 60:4403-4411.

13. Bacher U, Schnittger S, Kern W, et al.: Acute myeloid leukemia $(A M L)$ with $t(8 ; 21)(q 22 ; q 22)$ relapsing as AML with $\mathrm{t}(3 ; \mathbf{2 1})(\mathbf{q} 26 ; \mathbf{q} 22)$. Cancer Genet Cytogenet 2006, 168:172-174.

14.• van Rhenen A, Feller N, Kelder A, et al.: High stem cell frequency in acute myeloid leukemia at diagnosis predicts high minimal residual disease and poor survival. Clin Cancer Res 2005, 11:6520-6527.

A direct correlation was found between the frequency of CD34+CD38- cells at diagnosis and survival of AML patients.

This was mediated via the presence of MRD after chemotherapy.

15. Campana D: Minimal residual disease studies in acute leukemia. Am J Clin Pathol 2004, 122(Suppl):S47-S57.

16. Vidriales MB, Perez JJ, Lopez-Berges MC, et al.: Minimal residual disease in adolescent (older than 14 years) and adult acute lymphoblastic leukemias: early immunophenotypic evaluation has high clinical value. Blood 2003, 101:4695-4700.

17. Coustan-Smith E, Sancho J, Hancock ML, et al.: Clinical importance of minimal residual disease in childhood acute lymphoblastic leukemia. Blood 2000, 96:2691-2696.

18. van Dongen JJ, Seriu T, Panzer-Grumayer ER, et al.: Prognostic value of minimal residual disease in acute lymphoblastic leukaemia in childhood. Lancet 1998, 352:1731-1738.

19. Sievers EL, Lange BJ, Alonzo TA, et al.: Immunophenotypic evidence of leukemia after induction therapy predicts relapse: results from a prospective Children's Cancer Group study of 252 patients with acute myeloid leukemia. Blood 2003, 101:3398-3406.

20. Coustan-Smith E, Ribeiro RC, Rubnitz JE, et al.: Clinical significance of residual disease during treatment in childhood acute myeloid leukaemia. Br J Haematol 2003, 123:243-252.

21. San Miguel JF, Vidriales MB, Lopez-Berges C, et al.: Early immunophenotypical evaluation of minimal residual disease in acute myeloid leukemia identifies different patient risk groups and may contribute to postinduction treatment stratification. Blood 2001, 98:1746-1751.

22. Venditti A, Buccisano F, Del Poeta G, et al.: Level of minimal residual disease after consolidation therapy predicts outcome in acute myeloid leukemia. Blood 2000, 96:3948-3952.
23.• Feller N, van der Pol MA, van Stijn A, et al.: MRD parameters using immunophenotypic detection methods are highly reliable in predicting survival in acute myeloid leukaemia. Leukemia 2004, 18:1380-1390.

MRD frequency determined using multicolor flow cytometry was found to be a strong and independent risk factor for survival of patients with AML.

24. Kern W, Voskova D, Schoch C, et al.: Determination of relapse risk based on assessment of minimal residual disease during complete remission by multiparameter flow cytometry in unselected patients with acute myeloid leukemia. Blood 2004, 104:3078-3085.

MRD frequency determined using multicolor flow cytometry was found to be a strong and independent risk factor for survival of patients with AML.

25. Kern W, Schoch C, Haferlach T, Schnittger S: Monitoring of minimal residual disease in acute myeloid leukemia. Crit Rev Oncol Hematol 2005, 56:283-309.

26. Baer MR, Stewart CC, Dodge RK, et al.: High frequency of immunophenotype changes in acute myeloid leukemia at relapse: implications for residual disease detection (Cancer and Leukemia Group B Study 8361). Blood 2001, 97:3574-3580.

27. Kern W, Danhauser-Riedl S, Ratei R, et al.: Detection of minimal residual disease in unselected patients with acute myeloid leukemia using multiparameter flow cytometry for definition of leukemia-associated immunophenotypes and determination of their frequencies in normal bone marrow. Haematologica 2003, 88:646-653.

28. Roederer M, De Rosa S, Gerstein R, et al.: 8 color, 10parameter flow cytometry to elucidate complex leukocyte heterogeneity. Cytometry 1997, 29:328-339.

29. Voskova D, Schnittger S, Schoch C, et al.: Use of five-color staining improves the sensitivity of multiparameter flow cytomeric assessment of minimal residual disease in patients with acute myeloid leukemia. Leuk Lymphoma 2007, In press.

30. Schnittger S, Weisser M, Schoch C, et al.: New score predicting for prognosis in PML-RARA+, AML1-ETO+, or CBFBMYH11+ acute myeloid leukemia based on quantification of fusion transcripts. Blood 2003, 102:2746-2755.

31. Guerrasio A, Pilatrino C, De Micheli D, et al.: Assessment of minimal residual disease (MRD) in CBFbeta/MYH11positive acute myeloid leukemias by qualitative and quantitative RT-PCR amplification of fusion transcripts. Leukemia 2002, 16:1176-1181.

32. Gallagher RE, Yeap BY, Bi W, et al.: Quantitative real-time RT-PCR analysis of PML-RAR alpha mRNA in acute promyelocytic leukemia: assessment of prognostic significance in adult patients from intergroup protocol 0129. Blood 2003, 101:2521-2528.

33. Grimwade D, Walker H, Oliver F, et al.: The importance of diagnostic cytogenetics on outcome in AML: analysis of 1,612 patients entered into the MRC AML 10 trial. The Medical Research Council Adult and Children's Leukaemia Working Parties. Blood 1998, 92:2322-2333.

34. van Dongen JJ, Macintyre EA, Gabert JA, et al.: Standardized RT-PCR analysis of fusion gene transcripts from chromosome aberrations in acute leukemia for detection of minimal residual disease. Report of the BIOMED-1 Concerted Action: investigation of minimal residual disease in acute leukemia. Leukemia 1999, 13:1901-1928.

35. Schnittger S, Schoch C, Dugas M, et al.: Analysis of FLT3 length mutations in 1003 patients with acute myeloid leukemia: correlation to cytogenetics, FAB subtype, and prognosis in the AMLCG study and usefulness as a marker for the detection of minimal residual disease. Blood 2002, 100:59-66.

36. Cloos J, Goemans BF, Hess CJ, et al.: Stability and prognostic influence of FLT3 mutations in paired initial and relapsed AML samples. Leukemia 2006, 20:1217-1220.

37. Burmeister T, Marschalek R, Schneider B, et al.: Monitoring minimal residual disease by quantification of genomic chromosomal breakpoint sequences in acute leukemias with MLL aberrations. Leukemia 2006, 20:451-457. 
38. Falini B, Mecucci C, Tiacci E, et al.: Cytoplasmic nucleophosmin in acute myelogenous leukemia with a normal karyotype. N Engl J Med 2005, 352:254-266.

39. Gorello P, Cazzaniga G, Alberti F, et al.: Quantitative assessment of minimal residual disease in acute myeloid leukemia carrying nucleophosmin (NPM1) gene mutations. Leukemia 2006, 20:1103-1108.

40. Karakas T, Miething CC, Maurer U, et al.: The coexpression of the apoptosis-related genes $b c l-2$ and $w t 1$ in predicting survival in adult acute myeloid leukemia. Leukemia 2002, 16:846-854.

41. Weisser M, Kern W, Rauhut S, et al.: Prognostic impact of RT-PCR-based quantification of WT1 gene expression during MRD monitoring of acute myeloid leukemia. Leukemia 2005, 19:1416-1423.

42. Kreuzer KA, Saborowski A, Lupberger J, et al.: Fluorescent 5'-exonuclease assay for the absolute quantification of Wilms' tumour gene (WT1) mRNA: implications for monitoring human leukaemias. Br J Haematol 2001, 114:313-318.

43. Barjesteh van Waalwijk van Doorn-Khosrovani S, Erpelinck C, van Putten WL, et al.: High EVI1 expression predicts poor survival in acute myeloid leukemia: a study of 319 de novo AML patients. Blood 2003, 101:837-845.

44. Steinbach D, Hermann J, Viehmann S, et al.: Clinical implications of PRAME gene expression in childhood acute myeloid leukemia. Cancer Genet Cytogenet 2002, 133:118-123.

45. Paydas $\mathrm{S}$, Tanriverdi K, Yavuz $\mathrm{S}$, et al.: PRAME mRNA levels in cases with acute leukemia: clinical importance and future prospects. Am J Hematol 2005, 79:257-261.

46. Kerst G, Kreyenberg H, Roth C, et al.: Concurrent detection of minimal residual disease (MRD) in childhood acute lymphoblastic leukaemia by flow cytometry and real-time PCR. Br J Haematol 2005, 128:774-782.

47. Malec M, Bjorklund E, Soderhall S, et al.: Flow cytometry and allele-specific oligonucleotide PCR are equally effective in detection of minimal residual disease in ALL. Leukemia 2001, 15:716-727.

48. Perea G, Lasa A, Aventin A, et al.: Prognostic value of minimal residual disease (MRD) in acute myeloid leukemia (AML) with favorable cytogenetics $[t(8 ; 21)$ and inv $(16)]$. Leukemia 2006, 20:87-94.

49. Gianfaldoni G, Mannelli F, Baccini M, et al.: Clearance of leukaemic blasts from peripheral blood during standard induction treatment predicts the bone marrow response in acute myeloid leukaemia: a pilot study. Br J Haematol 2006, 134:54-57.

50. Goulden N, Virgo P, Grimwade D: Minimal residual disease directed therapy for childhood acute myeloid leukaemia: the time is now. Br J Haematol 2006, 134:273-282.

51. Leroy H, de Botton S, Grardel-Duflos N, et al.: Prognostic value of real-time quantitative PCR (RQ-PCR) in AML with $\mathrm{t}(8 ; 21)$. Leukemia 2005, 19:367-372.

52. Coustan-Smith E, Sancho J, Hancock ML, et al.: Use of peripheral blood instead of bone marrow to monitor residual disease in children with acute lymphoblastic leukemia. Blood 2002, 100:2399-2402.

53. van der Velden V, Jacobs DC, Wijkhuijs AJ, et al.: Minimal residual disease levels in bone marrow and peripheral blood are comparable in children with $\mathrm{T}$ cell acute lymphoblastic leukemia (ALL), but not in precursor-B-ALL. Leukemia 2002, 16:1432-1436.
54. Breccia M, Diverio D, Noguera NI, et al.: Clinico-biological features and outcome of acute promyelocytic leukemia patients with persistent polymerase chain reaction-detectable disease after the AIDA front-line induction and consolidation therapy. Haematologica 2004, 89:29-33.

55. Hess CJ, Feller N, Denkers F, et al.: Immunophenotypical minimal residual disease as a short term endpoint for monitoring effects of targeted inhibitors in acute myeloid leukemia [abstract]. Blood (ASH Annual Meeting Abstracts) 2005, 106:Abstract 545.

56. Jordan CT, Upchurch D, Szilvassy SJ, et al.: The interleukin-3 receptor alpha chain is a unique marker for human acute myelogenous leukemia stem cells. Leukemia 2000, 14:1777-1784.

57. Taussig DC, Pearce DJ, Simpson C, et al.: Hematopoietic stem cells express multiple myeloid markers: implications for the origin and targeted therapy of acute myeloid leukemia. Blood 2005, 4086-4092.

Hematopoietic stem cells obtained from normal bone marrow and cord blood were shown to express CD33 and CD123, similar to leukemic stem cells. This has important implications for targeted therapy against CD33 and CD123 targets.

58.• Bakker AB, Van den Oudenrijn S, Bakker AQ, et al.: C-type lectin-like molecule-1: a novel myeloid cell surface marker associated with acute myeloid leukemia. Cancer Res 2004, 64:8443-8450.

CLL-1 is expressed on the leukemic blasts at diagnosis, comparable to CD33 expression. As CLL-1 is not expressed on normal CD34+CD38-cells, CLL-1 might be used as a target for therapy in AML.

59. van Rhenen A, Feller N, Kelder A, et al.: The novel AML stem cell associated antigen CLL-1 discriminates between normal and leukemic stem cells [abstract]. Blood (ASH Annual Meeting Abstracts) 2005, 106:Abstract 4.

60. van Rhenen A, Feller N, Kelder A, et al.: In acute myeloid leukemia both malignant and normal stem cells can be detected in remission bone marrow [abstract]. Blood (ASH Annual Meeting Abstracts) 2006, 108:Abstract 2537.

61. Goodell MA, Brose K, Paradis G, et al.: Isolation and functional properties of murine hematopoietic stem cells that are replicating in vivo. J Exp Med 1996, 183:1797-1806.

62. Goodell MA, Rosenzweig M, Kim H, et al.: Dye efflux studies suggest that hematopoietic stem cells expressing low or undetectable levels of CD34 antigen exist in multiple species. Nat Med 1997, 3:1337-1345.

63. Wulf GG, Wang RY, Kuehnle I, et al.: A leukemic stem cell with intrinsic drug efflux capacity in acute myeloid leukemia. Blood 2001, 98:1166-1173.

64. Moshaver B, Feller N, Kelder A, et al.: The side population may provide the leukemic stem cell compartment complementary to the CD34+CD38- stem cell compartment. Implications for stem cell MRD detection and therapeutic targeting [abstract]. Haematologica 2006, 91:321.

65. Moshaver B, van der Pol MA, Westra GH, et al.: Identification of primitive subpopulations of acute myeloid leukemia side population (SP) stem cells defined by differentiation status and malignant character [abstract]. Blood (ASH Annual Meeting Abstracts) 2006, 108:Abstract 2538.

66. Moshaver B, van der Pol MA, Westra G, et al.: Acute myeloid leukemia remssion bone marrow reveals the presence of malignant and normal side population (SP) stem cells whose frequencies and ratios predict clinical outcome [abstract]. Blood (ASH Annual Meeting Abstracts) 2006, 108:Abstract 2314. 